\title{
On Bent Manifolds and Deformed Spaces
}

\author{
Shlomo Barak \\ Correspondence: Shlomo Barak, Taga Innovations 16 Beit Hillel St. Tel Aviv 67017 Israel. \\ E-mail: shlomo@tagapro.com
}

Received: May 19, 2019 Accepted: June 10, 2019 Online Published: July 1, 2019

doi:10.5539/jmr.v11n4p9 URL: https://doi.org/10.5539/jmr.v11n4p9

\begin{abstract}
Riemannian geometry is the geometry of bent manifolds. However, as this paper shows, it is also the geometry of deformed spaces. General Relativity (GR), based on Riemannian geometry, relates to space around the Sun and other masses as a bent 3D manifold.

Although a bent 3D space manifold in a 4D hyper-space is unimaginable, physicists accept this constraint. Our geometry of deformed spaces removes this constraint and shows that the Sun and other masses simply contract the 3D space around them. Thus, we are able to understand General Relativity (GR) almost intuitively - an intuition that inspires our imagination.

Space in GR is considered a continuous manifold, bent (curved) by energy/momentum. Both Einstein (1933) and Feynman (1963), considered the option of space being a deformed continuum rather than a bent (curved) continuous manifold. We, however, consider space to be a 3D deformed lattice rather than a bent continuous manifold. The geometry presented in this paper is the geometry of this kind of space.
\end{abstract}

Keywords: curvature, space, manifold, Riemannian geometry

\section{Introduction}

We relate to space not as a passive static arena for fields and particles but as an active elastic entity. The gravitational waves that General Relativity (GR) predicts, and have been observed recently, support our understanding. The mathematical objects of GR are n-dimensional manifolds in hyper-spaces with more dimensions than $\mathrm{n}$. These are not necessarily the physical objects that GR accounts for. Einstein was led to GR by arguments that were un-related to a possible elastic 3D space. However, in n-dimensional elastic deformed spaces, Euclidian geometry is not valid and we are compelled to use Riemannian geometry. Hence, GR is also the theory of our elastic 3D space, as Einstein (1933) and Feynman (1963) considered, and we are convinced (Barak 2017). Steane (2013) in his recent book "Relativity Made Relatively Easy" elaborates and clarifies these points. Rindler (2004) uses elastic spaces to enable visualization of bent manifolds, whereas Callahan (1999) declares: “...in physics we associate curvature with stretching rather than bending."

We define space density $\rho$, for an elastic space lattice, as the number of space cells per unit volume. The density of an un-deformed, uniform, space is denoted $\rho_{0}$. If space is uniform, all of its elementary cells are of the same size, and Euclidian geometry is valid. When the density is not uniform, i.e., cells are of different sizes, an internal observer discovers that Euclidian geometry is not valid. When this internal observer measures circles, they find an Excess Radius $\delta \mathrm{r}$ that differs from zero. This happens since the observer and their yardstick are both deformed exactly as the background.

We show that, at large, the geometry of bent manifolds and deformed spaces is similar. However, in certain cases they differ, and these un-noticed differences are sometimes the cause of perplexing situations in physics.

Note that the term Curvature and Radius of Curvature are used both for manifolds and spaces.

The following derived equation, (21) in Section 15, is the Gaussian Curvature $K$ at a point $P$ in a deformed space. $\rho$ is the space density at the point and $\nabla \rho$ is its gradient:

$$
\mathrm{K}=\frac{4 \pi}{45}\left(\frac{\nabla \rho}{\rho}\right)^{2}
$$

Sections 2 and 3 are introductory sections for those who are not familiar with differential geometry. 


\section{Intrinsic Curvature at a Point P on a 2D Manifold}

On a manifold a yardstick retains its length, but is bent like the manifold.

\subsection{D Curved Surfaces in a 3D Euclidian Space}

To obtain the curvature of a two-dimensional surface (manifold) at a point, $\mathrm{P}$, that is intrinsic to the surface, consider the length $\mathrm{C}$ of a closed path that is the locus of all points that are at the same geodesic distance, $\mathrm{r}$ from the point.

For a sphere of radius $\mathrm{r}_{0}$, the above circumference $\mathrm{C}$ for small $\mathrm{r} / \mathrm{r}_{0}$, as Figure 1 shows, is:

$$
\mathrm{C}=2 \pi \mathrm{r}_{0} \sin \left(\frac{\mathrm{r}}{\mathrm{r}_{0}}\right) \approx 2 \pi\left(\mathrm{r}-\frac{\mathrm{r}^{3}}{6 \mathrm{r}_{0}^{2}}\right)=2 \pi\left(\mathrm{r}-\frac{\mathrm{Kr}^{3}}{6}\right)
$$

Here $\mathrm{K}=\frac{1}{\mathrm{r}_{0}^{2}}$ is a natural definition for the curvature of a sphere of radius $\mathrm{r}_{0}$.

By extracting $\mathrm{K}$ from (1), we obtain the curvature $\mathrm{K}$ and re-define it for a more general case:

$$
\mathrm{K}=\frac{3}{\pi} \lim _{\mathrm{r} \rightarrow 0} \frac{2 \pi \mathrm{r}-\mathrm{C}}{\mathrm{r}^{3}}
$$

This $\mathrm{K}$ depends only on the intrinsic properties of the metric of the two-dimensional surface with no reference to the embedding space. The sign of the curvature is positive if $\mathrm{C}<2 \pi \mathrm{r}$, negative if $\mathrm{C}>2 \pi \mathrm{r}$, and zero if $\mathrm{C}=2 \pi$.

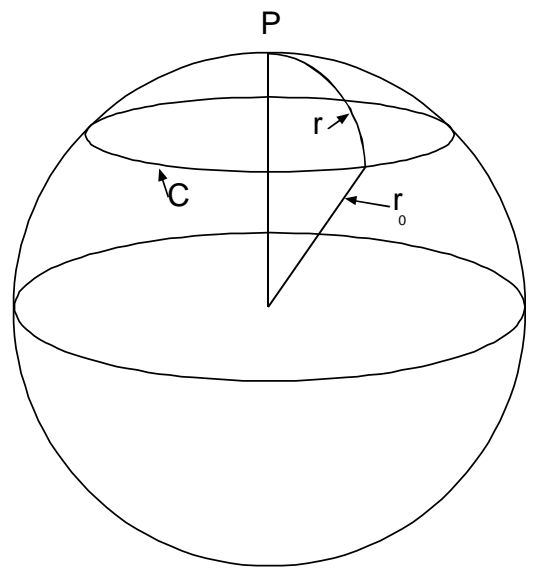

Figure 1. Curvature of a Sphere

Note that for a two-dimensional surface (manifold) Gaussian curvature can be defined as:

$$
\mathrm{K}=\frac{1}{\left(\mathrm{r}_{0}\right)_{1}\left(\mathrm{r}_{0}\right)_{2}}
$$

where $\left(\mathrm{r}_{0}\right)_{1}$ and $\left(\mathrm{r}_{0}\right)_{2}$ are the radii of the largest and smallest osculating circles of sections formed by the corresponding planes perpendicular to a third plane, tangential to the surface at $\mathrm{P}$. If the two osculating circles are on the same side of this third plane, we define the curvature as positive, and if on opposite sides - as negative. This definition ensures compatibility with the definition expressed by equation (2).

For a saddle surface, see Figure 3, the two osculating circles at point $\mathrm{P}$ in the middle of the saddle are on opposite sides of the third tangential plane to the surface at $\mathrm{P}$, and thus the saddle at $\mathrm{P}$ has a negative curvature.

\subsection{The Excess Radius or of Curved Manifolds}

The Excess Radius or of curved manifolds (Steane 2013), around a point P, see Figure 2. is:

$$
\delta \mathrm{r} \equiv \mathrm{r}_{\text {measured }}-\mathrm{c}_{\text {measured }} / 2 \pi \text {. }
$$




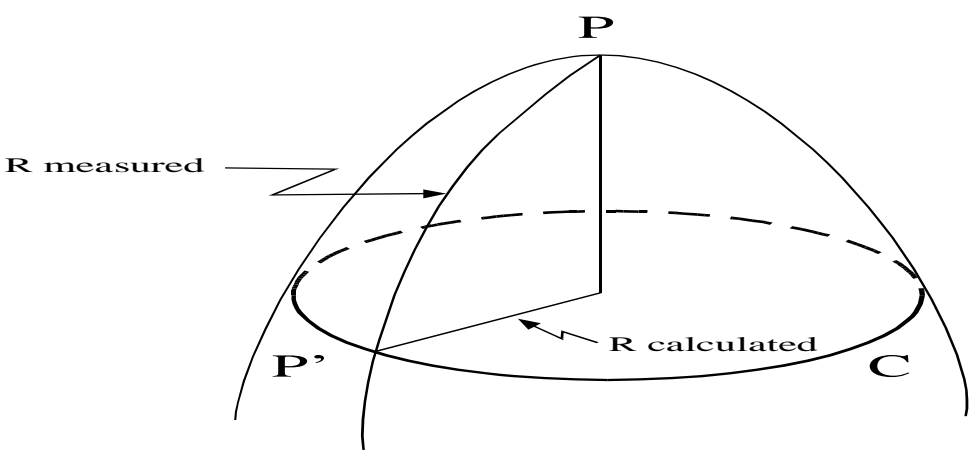

Figure 2. Excess Radius

Measuring distances with an intrinsic local yardstick gives the radius, $r$ (measured) from point $\mathrm{P}$ to point $\mathrm{P}^{\prime}$, and the circumference, $\mathrm{c}$ (measured), of a circle centered at $\mathrm{P}$ passing through $\mathrm{P}$.

The calculated radius is:

$$
\mathrm{r}_{\text {calculated }}=\mathrm{c}_{\text {measured }} / 2 \pi \text {. }
$$

\subsection{The Gaussian Curvature}

Here rmeasured is notated as simply r. According to (Steane 2013) equation (10.7), for a 2D bent surface (2D manifold) the Gaussian curvature is:

$$
\mathrm{K}=\lim _{\mathrm{r} \rightarrow 0} \frac{6 \delta \mathrm{r}}{\mathrm{r}^{3}}
$$

See the relation of equation (6) to equation (2):

$\mathrm{R}_{\mathrm{c}}$ is the radius of curvature at the point $\mathrm{P}$ on the surface, and:

$$
\mathrm{K}=1 / \mathrm{R}_{\mathrm{c}}{ }^{2}
$$

According to (Steane 2013), for the case of spherical symmetry, (6) for small $r$ is:

$$
\mathrm{K} \approx 6 \delta \mathrm{r} / \mathrm{r}^{3}
$$

For no radial symmetry, $\mathrm{K}$ is taken as the geometric mean (average) of the largest and smallest curvatures, $\mathrm{k} 1, \mathrm{k} 2$, one for each of the osculating circles; as if we have two separated surfaces.

2.4 Gaussian Curvature and the Schwarzschild Metric

Note (Bergman 1992) that, $R_{c}$, the radius of curvature of space in the Schwarzschild solution depends on the coordinate distance $r$ from the center of the mass $\mathrm{M}$, and is:

$$
\mathrm{R}_{\mathrm{c}}=\mathrm{r}_{\mathrm{s}}^{-1 / 2} \mathrm{r}^{3 / 2}
$$

where $r_{s}=2 \mathrm{GM} / \mathrm{c}^{2}$ is the Schwarzschild radius.

From (9) we get:

$$
\mathrm{K}=\mathrm{r}_{\mathrm{s}} / \mathrm{r}^{3}
$$

From (8) and (10) we get:

$$
\delta \mathrm{r} \sim 1 / 6 \mathrm{r}_{\mathrm{s}}
$$

More on the subject appears in Feynman (1963). 


\section{Intrinsic Negative Curvature at a Point $P$ on a 2D Manifold - A Saddle}

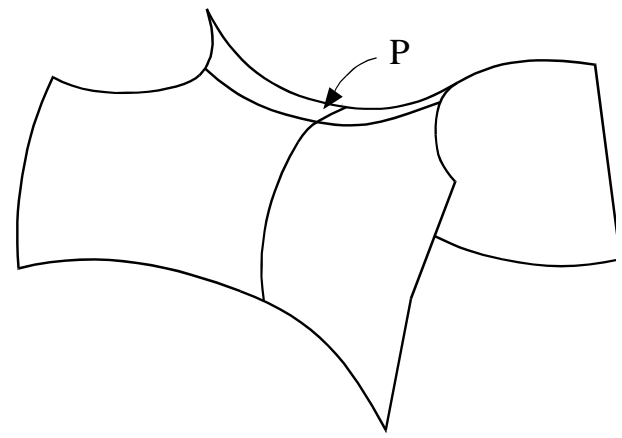

Figure 3. Saddle-like 2-D Manifold in 3-D Space

An osculating circle, of radius $r$, on the saddle around the point $P$ has a longer circumference than that of a circle on a flat surface, since the radius wiggles up and down (Rindler 2004). Therefore, according to (4) the Excess Radius $\delta \mathrm{r}$ of our curved manifold is negative. Gaussian curvature is:

$$
\mathrm{K}=\lim _{\mathrm{r} \rightarrow 0} \frac{6 \delta \mathrm{r}}{\mathrm{r}^{3}}
$$

and hence the saddle is curved negatively $\mathrm{K}<0$.

Let us draw a plane tangential to the saddle at P. Perpendicular to this plane; we draw two more planes with osculating circles through $\mathrm{P}$ from the two sides of the plane tangential to the saddle at P. Let $r_{\mathrm{c} 1}$ be the radius of an up circle with the smallest radius, and $r_{\mathrm{c} 2}$ be the radius of a down circle with, also, the smallest radius. In this case [like (3) but with a minus sign]:

If the radii are equal and denoted $r_{c}$ then:

$$
\mathrm{K}=-1 /\left(\mathrm{r}_{\mathrm{c} 1} \mathrm{r}_{\mathrm{c} 2}\right)
$$

$$
\mathrm{K}=-1 / \mathrm{r}_{\mathrm{c}}^{2}
$$

\section{Intrinsic Positive Curvature in a 2D Deformed Space}

In a deformed space a yardstick does not retain its length, but is contracted or dilated (stretched) like its local space.

\subsection{Positive Symmetric Curvature at a Point $P$}

Consider Figure 4, in which the circles represent space cells, or that a circle's diameter represents the length of a yardstick. Here, the yardstick at position $P$ is the smallest and from $P$ the yardstick increases in size symmetrically. This situation is analogous to a metallic plate, where the temperature increases from the center of the surface outwards, and thus the density decreases, i.e., the cell size increases. The 2D, inside observer, in Figure 4, finds that the ratio of the circumference $\mathrm{C}$ of the circle to the radius $\mathrm{r}$, as measured by the intrinsic yardstick, is:

$\mathrm{C} / \mathrm{r}<2 \pi$.

The Excess Radius $\delta$, in this case, is the same as for a curved manifold around a point P:

$$
\delta \mathrm{r} \equiv \mathrm{r}_{\text {measured }}-\mathrm{c}_{\text {measured }} / 2 \pi \text {. }
$$

For a positive curved 2D space, as Figure (3) shows, $\delta r=r_{\text {meas }}-r_{\text {cal }}>0$. 


$$
\begin{aligned}
& \mathrm{r}_{\text {measured }}=8 \\
& \mathrm{c}_{\text {measured }}=36 \\
& \mathrm{c} / \mathrm{r}=36 / 8=4.5<2 \pi \\
& \mathrm{r}_{\text {measured }}>\mathrm{r}_{\text {calculated }}
\end{aligned}
$$$$
\text { The Excess Radius }
$$$$
\delta \mathrm{r}=\mathrm{r}_{\text {meas }}-\mathrm{r}_{\mathrm{cal}}>0
$$

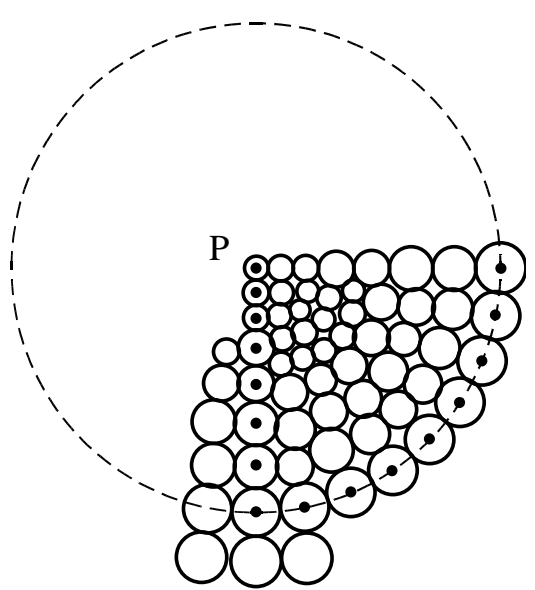

Figure 4. Positive Curvature

The 2D observer, therefore, concludes that there are two possibilities: they live in a two-dimensional space with a variable density, or on a curved two-dimensional surface "manifold" bent in a three-dimensional hyper-space.

The $2 \mathrm{D}$ observer cannot imagine a three-dimensional space, but may be able to accept the necessary abstraction. In any case, they are not able to decide between the two possibilities just by examining their locality.

\subsection{The Gaussian Curvature}

We define the Gaussian curvature in the same way as in Section 2.3:

$$
\begin{gathered}
K=\lim _{\mathrm{r} \rightarrow 0} \frac{6 \delta \mathrm{r}}{\mathrm{r}^{3}} \\
\mathrm{~K}=1 / \mathrm{R}_{\mathrm{c}}{ }^{2}
\end{gathered}
$$

$\mathrm{R}_{\mathrm{c}}$ is defined as the "radius of curvature" at the point P. Note, however, that the term "radius", in here, does not stand for the radius as measured by an internal or external observer.

According to Steane (2013), for the case of symmetry, (6) for small $r$ gives:

$$
\mathrm{K} \approx 6 \delta \mathrm{r} / \mathrm{r}^{3}
$$

\subsection{Asymmetric Positive Curvature at a Point $P$}

For no radial symmetry, $\mathrm{K}$ is taken as the geometric mean (average) of the largest and smallest curvatures, $\mathrm{k}_{1}, \mathrm{k}_{2}$; as if we have two surfaces with their corresponding excess radii

$\delta r_{1}$ and $\delta r_{2}$. A more accurate $\mathrm{K}$ should be taken based on the space density around $\mathrm{P}$.

\section{Intrinsic Negative Curvature in a 2D Deformed Space}

\subsection{Symmetric Curvature at a Point $P$}

$$
\begin{aligned}
& r_{\text {meas }}=8 \\
& C_{\text {meas }}=64 \\
& \frac{\mathrm{C}}{\mathrm{r}}=10.2>2 \pi \\
& \mathrm{r}_{\text {meas }}<\mathrm{r}_{\text {cal }}
\end{aligned}
$$

The Excess Radius

$\delta \mathrm{r}=\mathrm{r}_{\text {meas }}-\mathrm{r}_{\mathrm{cal}}<0$

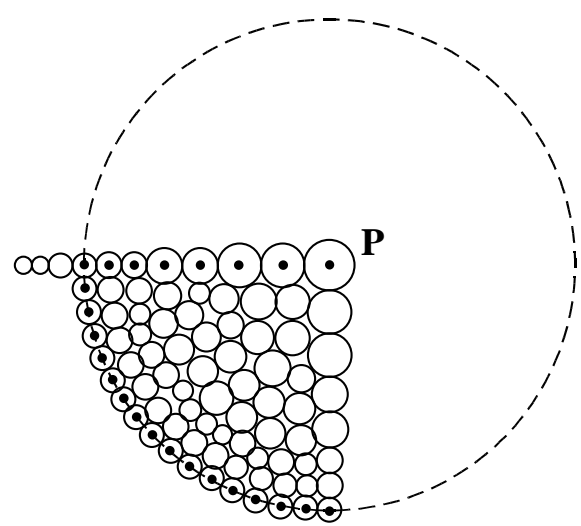

Figure 5. Negative Curvature 
Figure 5 shows a cell at point $\mathrm{P}$, which is the largest, and from $\mathrm{P}$ outwards the cells decrease in size. In this case, $\frac{\mathrm{C}}{\mathrm{r}}>2 \pi$ and $\delta \mathrm{r}=\mathrm{r}_{\text {meas }}-\mathrm{r}_{\text {cal }}<0$. In this case, according to (6):

The curvature is negative $\mathrm{K}<0$. Figure 4 is analogous to a metal plate, where the temperature decreases from the point $\mathrm{P}$ outwards, as if the point $\mathrm{P}$ is heated with a blow-torch.

\subsection{Asymmetric Curvature at a Point $P$}

For no radial symmetry, $\mathrm{K}$ is taken, with a minus sign, as the geometric mean (average) of the excess radii $\delta r_{1}$ and $\delta r_{2}$. An accurate $\mathrm{K}$ should be taken based on the space density around $\mathrm{P}$.

\section{Combined Curvature and Its Geometric Mean Radius}

A point $P$ is located in a zone of space, with an approximately symmetric radius of curvature $R_{L}$ at $P$. By the introduction of a mass $\mathrm{M}$ at $\mathrm{P}$, space is curved an additional curvature due to the presence of $\mathrm{M}$. Let this symmetric curvature have a radius RS. We can take the overall combined curvature at $\mathrm{P}$ as:

$$
\mathrm{K}=1 /\left(\mathrm{R}_{\mathrm{L}} \mathrm{R}_{\mathrm{S}}\right)
$$

The Geometric Mean Radius of the Combined Curvature is thus:

$$
R_{c}=\sqrt{ }\left(R_{L} R_{S}\right)
$$

This understanding is related to the long-standing issue of Dark Matter. In GR central acceleration is related to the curvature of space (Barak 2016). A star at point P curves space locally around it symmetrically. If the star is located at the skirt of a galaxy it is also exposed to the curvature of space around the galaxy, due to the non- homogeneous expansion of space around it, (Barak 2017). This exposure contributes an additional general central acceleration, wrongly interpreted as due to the presence of additional mater - Dark Mater. The geometric mean of the compound local and general accelerations, which is the Milgrom phenomenological equation (Barak 2017), is related to the Geometric Mean Radius of the Compound Curvature.

\section{Examples of Curvature in a 2D Deformed Space}

\subsection{Positive Curvature}

To measure circles around a point $P$, Figure 3 , we take the normalized density at $P$ as $\rho(0)=1$. Assuming radial symmetry, the density for external observers is $\rho(r)$. Let $d r$ ' be the yardstick's length for an internal observer and dr for the external observer. These dr' and dr are related by:

$\mathrm{dr}{ }^{\prime}=\mathrm{dr} / \rho(\mathrm{r})$.

At $P$ these yardsticks are equal since we have chosen $\rho(0)=1$.

If, at a distance $R$ from $P$ the density is $\rho(R)=1 / 2$, then:

$\mathrm{dr} r^{\prime}=\mathrm{dr} / \rho(\mathrm{R})=2 \mathrm{dr}$

For external observers the circumference of a circle with radius $\mathrm{R}$ is $\mathrm{c}=2 \pi \mathrm{R}$, whereas for internal observers it is:

$c^{\prime}=2 \pi R\left(d r / d r^{\prime}\right)_{\text {atR }}=2 \pi R \rho(R)=\pi R$.

The radius of this circle, as measured by internal observers, is:

$\mathrm{R}^{\prime}=\int_{0}^{R} \mathrm{dr}^{\prime}=\int_{0}^{R} 1 / \rho(\mathrm{r}) \cdot \mathrm{dr}$

and it should be larger than R. If, for example:

$\rho(r)=1 /\left(1+r / r_{0}\right)=1 /(1+r / 18)$

then indeed $\rho(0)=1$ and, for $R=r=9, \quad \rho(R)=1 / 2$. The radius $R^{\prime}$ is then:

$\mathrm{R}^{\prime}=\int_{0}^{\mathrm{R}}(1+\mathrm{r} / 18) \cdot \mathrm{dr}=\mid\left(\mathrm{r}+\mathrm{r}^{2} /\left.36\right|_{0} ^{\mathrm{R}}=11.25\right.$

Thus the Excess Radius is:

$\delta \mathrm{R}^{\prime}=\mathrm{R}^{\prime}-\mathrm{c}^{\prime} / 2 \pi=\mathrm{R}^{\prime}-1 / 2 \mathrm{R} \sim 11-4.5 \sim 6.5$.

This criterion, $\delta \mathrm{R}^{\prime}>0$, for positive curvature, expresses the fact that space around $\mathrm{P}$ is contracted. 


\subsection{Negative Curvature}

If, at a distance R from the origin P, see Figure (4), the density is $\rho(R)=2$, then:

$\mathrm{dr} r^{\prime}=\mathrm{dr} / \rho(\mathrm{R})=1 / 2 \mathrm{dr}$

For external observers the circumference of a circle of radius $R$ is $c=2 \pi R$, whereas for the internal observers it is:

$c^{\prime}=2 \pi R(d r / d r ')_{a t R}=2 \pi R \rho(R)=4 \pi R$

The radius of this circle, as measured by the internal observer, is:

$\mathrm{R}^{\prime}=\int_{0}^{R} \mathrm{dr}^{\prime}=\int_{0}^{R} 1 / \rho(\mathrm{r}) \cdot \mathrm{dr}$

and it should be smaller than R. If, for example:

$\rho(r)=\left(1+r / r_{0}\right)=(1+r / 9)$

then indeed $\rho(0)=1$, and for $R=r=9$ we get $\rho(R)=2$. The radius $R^{\prime}$ is then:

$\mathrm{R}^{\prime}=\int_{0}^{\mathrm{R}} 1 /(1+\mathrm{r} / 9) \cdot \mathrm{dr}=|9 \ln (1+\mathrm{r} / 9)|_{0}^{\mathrm{R}}=6.24$

Thus the Excess Radius, in this case, is:

$\delta \mathrm{R}^{\prime}=\mathrm{R}^{\prime}-\mathrm{c}^{\prime} / 2 \pi=\mathrm{R}-2 \mathrm{R}=6.24-9 \sim-2.8$.

This criterion, $\delta R^{\prime}<0$, for negative curvature, expresses the fact that space around $p$ is dilated.

\section{A Saddle}

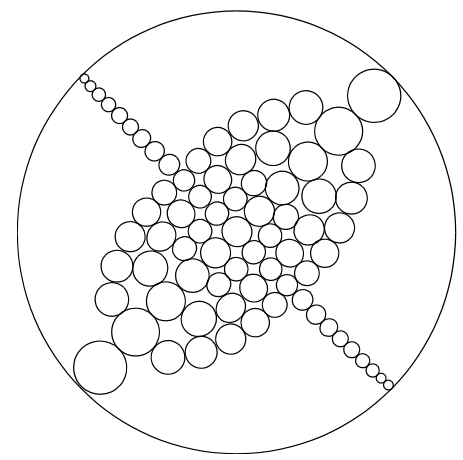

Figure 6. Saddle-like Elastic 2-D Space

At point $P$ in the center, see Figure 6, in 1/4 $\pi$ direction, the outward dilation of space means that the curvature is positive, with a radius of curvature $\mathrm{R}+$. In the $3 / 4 \pi$ direction the outward contraction of space means that the curvature is negative, with a radius of curvature $\mathrm{R}$-.

Thus, we can define the overall curvature $\mathrm{K}$ as:

$$
\mathrm{K}=1 /\left(\mathrm{R}_{+} \mathrm{R}_{-}\right)
$$

If $\mathrm{R}_{+}-\mathrm{R}_{-}>0$ then approximately $\mathrm{K}>0$, and if $\mathrm{R}_{+}-\mathrm{R}_{-}<0$ then $\mathrm{K}<0$.

Note that an accurate definition is related to space density in the all area.

\section{Intrinsic Curvature at a Point $P$ in a 3D Deformed Space}

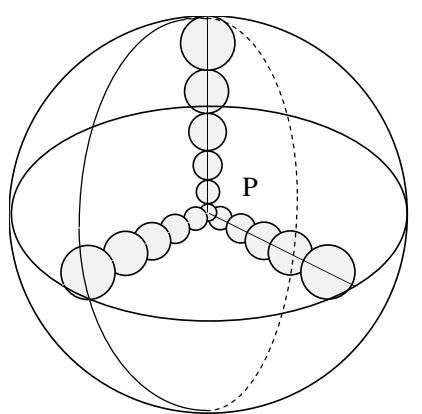

Figure 7. Three-dimensional Positive Curvature

Figure 7 shows a small cell located at point $\mathrm{P}$, and cells of increasing size radiating from $\mathrm{P}$ with spherical symmetry. If we 
measure the circumference of a great circle whose center is $\mathrm{P}$, in any direction, a measurement of the Excess Radius yields:

$$
\mathrm{r}=\mathrm{r}_{\text {meas }}-\mathrm{r}_{\text {cal }}>0
$$

Measurements of circumferences and radii can be taken around any point inside the sphere. But for any point, except point $\mathrm{P}$ in Figure 7, there is no symmetry.

For a deformed 3D space with spherical symmetry around $\mathrm{P}$ the Gaussian curvature at $\mathrm{P}$ is also expressed by equation (2).

For the case of no symmetry, we have to determine the degree of deformation of circles around point $\mathrm{P}$, in three orthogonal planes through $\mathrm{P}$. For each of the orthogonal planes we have to determine the largest and smallest curvatures, $\mathrm{k}_{1}, \mathrm{k}_{2}$, called the principle curvatures. Thus to specify a deformed three-dimensional space around a point $\mathrm{P}$ we need $3 \times 2$ numbers. The average of these six principle curvatures is the average Gaussian curvature of the deformed space at the locality of $\mathrm{P}$.

According to the above, a complete definition of curvature in close proximity to a point in three-dimensional space requires six "curvature numbers". These represent three pairs of curvature numbers for each of the three intersecting planes perpendicular to each other. These curvature numbers are components of a symmetric tensor of $2^{\text {nd }}$ rank called the contracted Riemannian tensor of curvature, or the Ricci tensor.

\subsection{Geodesics}

Figure 8 shows the shortest distance between two points, A and B, in a deformed two-dimensional space. This figure shows that the shortest distance between points A and B for an internal observer, with their changing yardstick, is the solid line path that passes through the centers of seven cells, and not the "straight" dashed line through A and B, that passes through nine cells.

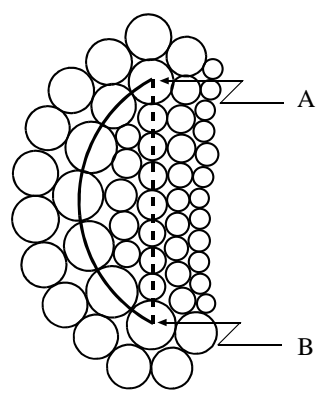

Figure 8. Geodesics

The shortest distance between points A and B, solid line, is the geodesic line. There is no difficulty in imagining this in three dimensions.

\section{Local Versus Global Curvature and the Flat Universe}

If a measurement of the radius and circumference of a circle at any point, on a surface, produces the Excess Radius result $\delta r=r_{\text {meas }}-r_{\text {cal }}>0$, the observers will conclude that they live on a finite two-dimensional closed surface in a three-dimensional space, and not in a deformed space. Thus we realize that curving and deforming are equivalent only locally.

If space density of the universe is, by and large, uniform, and only changes locally around stars and galaxies, then on the scale of the universe, Euclidian geometry is valid. In this case, we can say that we are living in a Flat Universe. In other words, our universe is curved only locally, but not globally. According to observations made by cosmologists our universe is flat. Cosmologists assume that the universe is a $3 \mathrm{D}$ manifold bent in a hyper-space with an extra dimension. Hence, they wonder why, from all possible values, the curvature of the universe happens to be zero.

There is no need to wonder; if we consider ourselves 3D internal observers in a universe whose space is 3D and elastic, with local deformations only. In our motion from place to place, both ourselves and our yardstick change. The only way for us to learn about our deformed space is thus to use Riemannian geometry. We are part of some cellular design and, in motion, it is the design and the energy that moves, not the cells. This is possible if elementary particles are not alien to space but simply wavepackets in motion. 


\section{Infinite Closed Space}

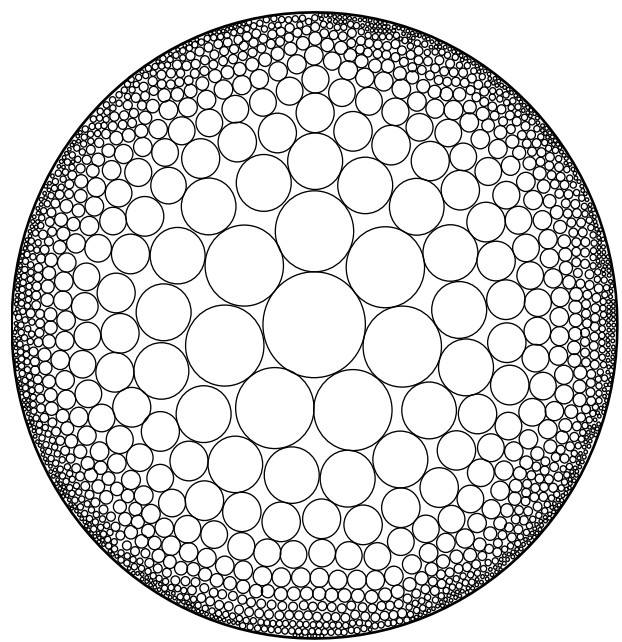

Figure 9. Infinite Closed Space

At first sight, space that is both infinite and closed seems a contradiction, but this is not so. Figure 9 shows, for example, a closed two-dimensional space in a circle of radius $\mathrm{R}$, at whose center is a cell of finite size. From this center outwards, the cell size decreases, tending towards zero as they approach the circumference of the circle. In this case, the number of cells can be infinite, while the area they occupy, the area of a circle of radius $\mathrm{R}$, is finite.

Obviously, this reasoning can also be applied to a three-dimensional sphere, and many other examples of an infinite closed space exist.

\section{The Metric, Ricci Tensor and General Relativity (GR)}

The metric tensor $\mathrm{g}_{\mathrm{ij}}$ and Ricci tensor $\mathrm{R}_{\mathrm{ij}}$ appear in the left-hand side of the Einstein equation of GR (Rindler 2004):

$$
\mathrm{R}_{\mathrm{ij}}-\frac{1}{2} \mathrm{Rg}_{\mathrm{ij}}=8 \pi \mathrm{G} / \mathrm{c}^{4} \cdot \mathrm{T}_{\mathrm{ij}}
$$

A metric tensor represents local deformations, around a given point, of both a continuum and a lattice. For a lattice, this representation is legitimate only if the sizes of its cells are orders of magnitude smaller than the scale of the deformation in this locality since we are using continuous differential equations. An example of such a metric is the Schwarzschild metric (Rindler 2004). A metric tensor can also represent a global deformation like the FRW metric (Rindler 2004).

The Ricci curvature tensor is the corresponding matrix of traces of the Riemannian curvature tensor. An element $R_{i j}$ of the Ricci tensor is an average of sectional curvatures, around a given point, of the intersection line of a manifold with the $\mathrm{x}_{\mathrm{i}} \mathrm{x}_{\mathrm{j}}$ plane. The scalar curvature $\mathrm{R}$ is defined as the trace of the Ricci curvature.

The reason why Einstein added the term $-\frac{1}{2} \operatorname{Rg}_{\mathrm{ij}}$ to his equation is explained by Feynman (1963) and Penrose (2004) pages $461-2$.

\section{4D Deformed Spacetime and Riemannian Geometry}

To an outside observer, the universe acts to all intents and purposes as an elastic body and the theory of elasticity is the tool with which to perform measurements and calculations. For an internal observer, in the universe, rulers, clocks and they themselves change according to the deformation (space density) at any particular point. This is a change that the internal observer cannot detect directly. The only way an internal observer can detect the distortion, i.e., the change in space density, is by measuring triangles and circles, for example, and detecting any deviation in the sum of the angles in a triangle that should be $180^{\circ}$ in undistorted space, or from the ratio of the circumference of a circle to its radius that should be $2 \pi$ in undistorted space. The internal observer interprets any deviation as invalidity of Euclidian geometry, whereas the external observer sees the deviation simply as an expression of a change in space density.

Apparently, the only geometry that the internal observer can adopt in the above case where rulers and clocks change, but do not appear to change, is Riemannian geometry.

Clearly, from the above, by adopting the point of view of the external observer it is possible to build a simple and tangible picture of the universe. 
This tangible picture relates only to distorted three-dimensional space, which is therefore seen as curved, but, because this distortion determines the speed of light, it anyway determines the rate of clocks at any particular point. Thus, in finding a solution to any dynamic problem of the motion of a beam of light, or a particle, we must deal with an abstract mathematical space with four dimensions. Here we can certainly dispense with the need for tangibility because this is not a real space, but a mathematical space that we build for the purpose of calculations.

\section{Curvature in Deformed Spaces Is a Function of Space Density}

\subsection{Morgan (1998): On Riemannian Geometry}

"Remark. An intrinsic definition of the scalar curvature $\boldsymbol{R}$ at a point $\boldsymbol{p}$ in an m-dimensional surface $S$ could be based on the formula for the volume of a ball of intrinsic radius $r$ about $p$ :

$$
\text { volume }=\alpha_{\mathrm{m}} \mathrm{r}^{\mathrm{m}}-\alpha_{\mathrm{m}} \frac{\mathrm{R}}{3 \cdot(\mathrm{m}+2)} \mathrm{r}^{\mathrm{m}+2}+\ldots,
$$

where ${ }_{m}$ is the volume of a unit ball in $R^{m}$. When $m=2$, this formula reduces to Equation (3.8). The analogous formula for spheres played a role in $R$. Schoen's solution of the Yamabe problem of finding a conformal deformation of a given Riemannian metric to one of constant scalar curvature (see Schoen [Sch, Lemma 2])." This Lemma is presented by Bray and Minicozzi (2018).

From equation (6.10), for any dimension, we can obtain the approximated scalar curvature of two-dimensional space (16) and that of three-dimensional space (17):

Note that we notate the scalar curvature $\mathrm{R}$, at a point $\mathrm{P}$ in the quotation (6.10), by the letter K. Note also that the term, volume, in (6.10) relates to a n-dimensional volume.

For a 2D space:

$$
\mathrm{K}=\frac{12}{\pi}\left\{\frac{\pi \mathrm{r}^{2}-\mathrm{S}}{\mathrm{r}^{4}}\right\}
$$

And for a 3D space:

$$
\mathrm{K}=\frac{45}{4 \pi}\left\{\frac{\frac{4 \pi}{3} \mathrm{r}^{3}-\mathrm{V}}{\mathrm{r}^{5}}\right\}
$$

14.2 Space Density and the Volume Change

The relative change in space density is: $\frac{\delta \rho}{\rho}$

and the relative volume change is: $\frac{\delta \mathrm{V}}{\mathrm{V}} \quad$ but: $\rho \propto \frac{1}{\mathrm{~V}}$

and therefore: $\frac{\delta \rho}{\rho}=\frac{\delta \frac{1}{\mathrm{~V}}}{\frac{1}{\mathrm{~V}}}=\frac{-\frac{\delta \mathrm{V}}{\mathrm{V}^{2}}}{\frac{1}{\mathrm{~V}}}=-\frac{\delta \mathrm{V}}{\mathrm{V}} \quad$ and therefore:

$$
\frac{\nabla \rho}{\rho}=-\frac{\nabla \mathrm{V}}{\mathrm{V}}
$$

In the case of spherical symmetry: $\frac{\nabla \rho}{\rho}=-\frac{\frac{\partial V}{\partial r}}{V} \frac{\mathbf{r}}{r}$

\section{The Scalar Curvature $K$ and the Space Density $\rho(r)$}

This section integrates the results of the previous sections to reach the goal of relating Curvature K to Space Density $\rho(\mathrm{r})$. The scalar Riemannian curvature in a three-dimensional space (17) is: 


$$
\mathrm{K}=\frac{\frac{4 \pi}{3} \mathrm{r}^{3}-(\text { Volume of Intrinsic Radius })}{5 \mathrm{r}^{5}}
$$

For the spherical symmetric case:

$\mathrm{K}=\frac{\frac{4 \pi}{3} r^{3}-\frac{4 \pi}{3}(r-u)^{3}}{5 r^{5}} \cong \frac{4 \pi}{5} \frac{u}{r^{3}}$ and for the simple case $u=c r:$

$$
\mathrm{K}=\frac{\frac{4 \pi}{3} \mathrm{r}^{3}-\frac{4 \pi}{3}(1-c)^{3} \mathrm{r}^{3}}{5 \mathrm{r}^{5}} \cong \frac{\frac{4 \pi}{3} 3 \mathrm{c}^{2} \mathrm{r}^{3}}{5 \mathrm{r}^{5}}=\frac{4 \pi}{5} \frac{\mathrm{c}^{2}}{\mathrm{r}^{2}}
$$

On the other hand:

$\frac{\rho_{0}-\rho}{\rho}=\frac{\mathrm{V}_{0}-\mathrm{V}}{\mathrm{V}}=\frac{(\mathrm{r}+\mathrm{u})^{3}-\mathrm{r}^{3}}{(\mathrm{r}+\mathrm{u})^{3}} \cong 3 \frac{\mathrm{u}}{\mathrm{r}}$

$\frac{\nabla \rho}{\rho}=3 \frac{\nabla u}{r}$ For the spherical symmetric case: $\frac{\frac{\partial \rho}{\partial r}}{\rho}=3 \frac{\frac{\partial u}{r}}{r}$ and if:

$\mathrm{u}=\mathrm{cr}: \frac{\frac{\partial \rho}{\partial r}}{\rho}=\frac{3 \mathrm{c}}{\mathrm{r}} \quad$ then:

$$
\left(\frac{\nabla \rho}{\rho}\right)^{2}=9 \frac{\mathrm{c}^{2}}{\mathrm{r}^{2}}
$$

Comparing (19) to (20) gives for 3D:

$$
K=\frac{4 \pi}{45}\left(\frac{\nabla \rho}{\rho}\right)^{2}
$$

The scalar curvature, $K$, as a function of space density $\rho(r)$, only.

\section{Summary}

Our geometry enables us to visualize the various solutions of the General Relativity field equation. This is made possible since, instead of the Riemannian geometry of bent manifolds in higher dimensions, we relate to deformed spaces using the same mathematical terminology and formalism.

Tangibility inspires imagination. "Imagination is more important than knowledge" A. Einstein.

\section{Acknowledgments}

We would like to thank Mr. Roger M. Kaye for his linguistic contribution and technical assistance.

\section{References}

Barak. S. (2017). A Theoretical Derivation of the Milgrom MOND Equation hal-01471151v3.

Barak. S. (2017). The Geometrodynamics of Space.

Barak. S. (2016). On the Essence of Gravitation and Inertia, Part 2: The Curving of Space by an Elementary Particle hal-01405460 (2016).

Bergman. P. G. (1992). The Riddle of Gravitation Dover p.195.

Bray, H. L., \& Minicozzi II, W. P. (2018). The Mathematics of Richard Schoen, Notices of the AMS p.p.1352-1355 Dec.

Callahan. J. J. (1999). The Geometry of Spacetime, Springer. 
Einstein. A. (1933). Ideas and Opinions, Wings Books NY, “On the Method...”.

Feynman. R. (1963). Lectures on Gravitation, Addison Wesley.

Feynman. R. (1963). Six Not So Easy Pieces, Addison Wesley.

Morgan. F. (1998). Riemannian geometry, A.K. Peters.

Penrose. R. (2004). The Road to Reality. Knopf pages 461-2

Rindler. W. (2004). Relativity Oxford

Steane. A. M. (2013). Relativity made relatively easy. Oxford.

\section{Copyrights}

Copyright for this article is retained by the author(s), with first publication rights granted to the journal.

This is an open-access article distributed under the terms and conditions of the Creative Commons Attribution license (http://creativecommons.org/licenses/by/4.0/). 